\title{
The dependence of the radiation damage formation on the substrate implant temperature in GaN during $\mathrm{Mg}$ ion implantation
}

\author{
S. Whelan ${ }^{a)}$ and M. J. Kelly \\ Department of Engineering, University of Cambridge, Trumpington St., Cambridge CB2 1PZ, \\ United Kingdom \\ R. Gwilliam and C. Jeynes \\ Advanced Technology Institute, University of Surrey, Guildford GU2 7XH, United Kingdom \\ C. Bongiorno \\ Consiglio Nazionale delle Ricerche Istituto per la Microelettromica ei Microsistemi (CNR-IMM) Stradale \\ Primosole 50, 95121 Catania, Italy
}

(Received 4 January 2005; accepted 2 May 2005; published online 6 July 2005)

\begin{abstract}
In pursuit of $p$-type doping, we have implanted $\mathrm{GaN}$ with $\mathrm{Mg}$ ions at 200 and $500 \mathrm{keV}$ with the substrate temperature maintained at -150 (cold) or $+300{ }^{\circ} \mathrm{C}$ (hot) during ion irradiation. The samples have been annealed at $1000{ }^{\circ} \mathrm{C}$ postion implantation. The radiation damage peak position (and its profile), the dopant distribution, and the damage stability during annealing were all shown to be dependent upon the GaN substrate temperature during implantation. The damage peak position in the solid was reduced for cold implantation. The dopant distribution in the solid depends upon the implant temperature and in agreement with the damage measurements, the $\mathrm{Mg}$ range is shallower in $\mathrm{GaN}$ for cold implants when compared to hot implants. The trends observed suggest that the dynamic defect annealing rate during irradiation is reduced for cold implantation, and the subsequent increase in the damage level (scattering centers) formed during the damage buildup reduces the ion range in the solid. In turn, the reduced ion range subsequently limits the final damage range. The rate of damage removal during thermal annealing in the samples implanted at cold temperature was increased: this is explained by the greater complexity of defects caused during high-temperature implantation, due to the raised level of dynamic defect annealing. () 2005 American Institute of Physics. [DOI: 10.1063/1.1940142]
\end{abstract}

\section{INTRODUCTION}

Ion implantation is the process of choice for doping in electronic device manufacture because of the selected area capability and the flexibility offered over implant species, dose, and range. However, efficient $p$-type doping of $\mathrm{GaN}$ through implantation has been problematic to date because of the complex nature of the as-grown defects in $\mathrm{GaN}$, and the defects that are introduced during ion irradiation. A full review ${ }^{1}$ has summarized the crystal defects that are associated with the activation process of acceptor atoms in $\mathrm{GaN}$, and will not be discussed here. An understanding of the implant radiation damage formation process in the $\mathrm{GaN}$ matrix and its removal during thermal processing is required if the full potential of selective area $p$-type doping in $\mathrm{GaN}$ is to be achieved. These issues will be addressed in this paper.

It has been previously demonstrated that the temperature of the semiconductor substrate during ion irradiation affects the formation of radiation damage in the solid. It has been shown that for the case of $\mathrm{Si}$, the sample temperature affects the damage creation process, ${ }^{2}$ and ultimately the defectdriven dopant diffusion during high-temperature annealing. It was observed with ion channeling experiments that the damage formation was reduced in samples implanted at an elevated temperature, and that the damage annealing at the

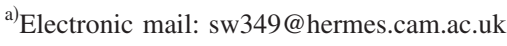

end of the defect range was not as efficient as in samples implanted at cold temperatures during postimplant annealing. The effect of the substrate temperature upon the damage formation during implantation in GaAs has also been investigated by many groups. Although the majority of the work focused upon implantation for isolation (and not doping), ${ }^{3}$ the trends observed suggest that the damage formed during elevated temperature implantation is more stable. Previous work on GaN (Ref. 4) proposed that a more stable implant damage was formed for elevated temperature implants, in line with $\mathrm{Si}$ and $\mathrm{GaAs}$, and suggested that lattice expansion occurred during cold implantation. It is proposed here that an improved understanding of the processes that occur as a function of the implant temperature (equivalently a function of the dynamic annealing) will enhance our knowledge and understanding of the damage formation processes in GaN.

We have implanted GaN with $\mathrm{Mg}$ ions at energies of 200 and $500 \mathrm{keV}$ at substrate temperatures of -150 (cold) and $+300{ }^{\circ} \mathrm{C}$ (hot). For the samples implanted at cold temperatures, an increase in the Ga disorder is observed in the surface region and the damage peak position is reduced in the solid when compared to implants performed at elevated temperatures. The secondary-ion-mass spectrometry (SIMS) profiling of $\mathrm{Mg}$ in the $\mathrm{GaN}$ provides clear evidence that the dopant distribution in the solid also depends upon the implant temperature and it is observed that $\mathrm{Mg}$ has a shorter range in $\mathrm{GaN}$ for cold implants. The damage formed in a 
sample implanted at cold temperatures is shown to be less stable than the damage formed in samples implanted at elevated temperatures during thermal annealing.

\section{EXPERIMENTAL METHODS}

$\mathrm{Mg}$ ion implantation into low-doped $n$-type $\mathrm{GaN}$ (for subsequent $p$-type doping) has been performed with a Van de Graff accelerator at ion energies of 200 and $500 \mathrm{keV}$ to doses of $1.1 \mathrm{E} 15$ and $1.4 \mathrm{E} 15 \mathrm{ions} / \mathrm{cm}^{2}$, respectively, and at substrate temperatures of -150 (cold) and $+300{ }^{\circ} \mathrm{C}$ (hot). The implant doses have been chosen so that the amount of damage formed within the region of the damage peak is a constant irrespective of the implant energy, as inferred by TRIM simulations. ${ }^{5}$ After implantation, the samples have been subjected to a rapid thermal anneal (RTA) at a temperature of $1000{ }^{\circ} \mathrm{C}$ for $20 \mathrm{~s}$ in a $\mathrm{N}_{2}$ ambient. In order to avoid decomposition, the proximity method, ${ }^{6}$ whereby a $\mathrm{GaN}$ wafer is placed upon the surface of the sample of interest, was used. No evidence of decomposition was observed with this technique.

The damage profiles following implantation and annealing have been obtained with Rutherford backscattering spectroscopy $^{7}$ (RBS) channelling measurements. RBS was performed with a MV Tandetron accelerator using 3-MeV He giving a depth resolution of $25 \mathrm{~nm}$. Data analysis of the raw RBS data utilized the IBA DataFurnace ${ }^{8}$ which fits the data with elemental profiles and then converts the data from backscattered ions versus ion energy (channel number) into $\mathrm{Ga}$ disorder versus depth. The $\mathrm{Mg}$ profile in $\mathrm{GaN}$ has been measured by SIMS with a Cameca IMS3F machine, using $\mathrm{O}_{2}^{+}$ primary ion $(10 \mathrm{keV})$ bombardment with positive-ion detection. In order to eliminate charging effects on the $\mathrm{GaN}$, a thin layer of $\mathrm{Au}$ was deposited on the sample surface prior to SIMS and an electron flood gun was used in order to maintain charge neutrality. Dark-field cross-section transmission electron microscopy (XTEM) has been performed upon selected samples using a Jeol JEM 2010 with an acceleration voltage of $200 \mathrm{kV}$. The images were taken in a two-beam condition with the sample tilted around the surface direction in order to increase the contrast due to the lattice distortion, without spreading in the surface projection. The sample cross section was performed with mechanical polishing and the final step was performed with a Gatan precision ion polishing system (PIPS) using 5-kV $\mathrm{Ar}^{+}$ions at $6^{\circ}$.

\section{RESULTS}

We now present and interpret the structural, chemical, and theoretical data obtained from $\mathrm{Mg}$ ion-implanted (and annealed) GaN. All analysis techniques employed for this investigation have been performed at room temperature.

RBS analysis provides an indication of the amount of displaced interstitial $\mathrm{Ga}$ in the solid, and is therefore used as a measure for the implant damage. The RBS results from as-implanted samples are presented in Fig. 1 . The Mg doses were chosen so that the peak damage levels are approximately constant (2.5 at. \%), and hence the differences observed are more likely to be implant temperature dependent and not dose dependent (however, this may not be strictly

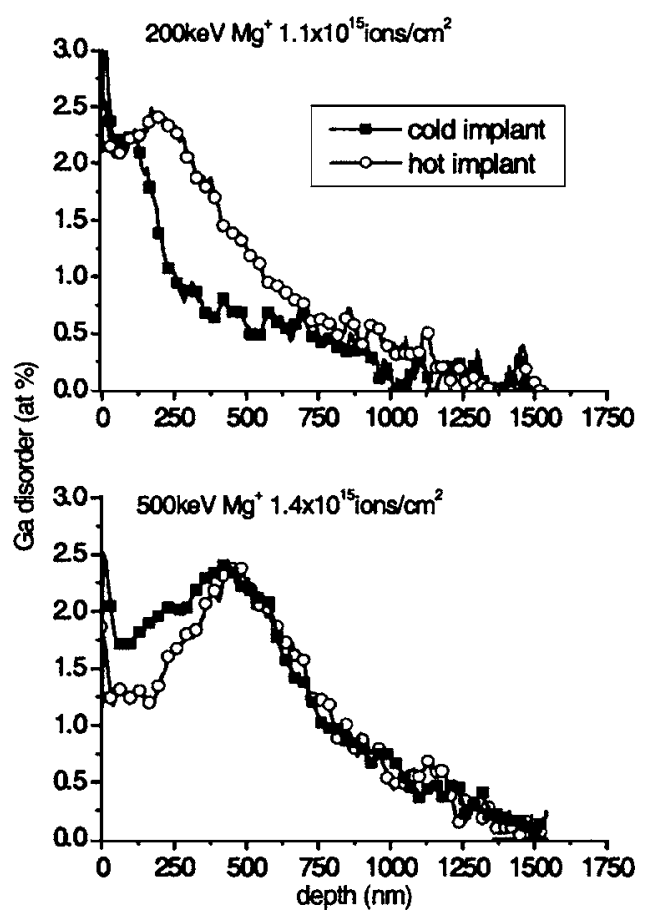

FIG. 1. Rutherford backscattering spectra (converted onto a depth scale with the IBA DataFurnace) obtained from GaN samples implanted with $\mathrm{Mg}$ ions at different energies and different temperatures.

true). The damage profile extends further into the solid for higher-energy implants as expected, and it can be seen that the shape and the peak position of the damage profile (displaced $\mathrm{Ga}$ ) depend upon the implant substrate temperature.

For the implant energies investigated here, the position of the damage peak is reduced in depth for the cold implants. The extent of the reduction appears to depend upon the implant energy. For 200-keV implantation, the difference in the damage peak position as a function of the implant temperature is larger (105 nm for cold and $180 \mathrm{~nm}$ for hot implants) and much more evident than for the 500-keV implant (420 nm for cold and $445 \mathrm{~nm}$ for hot implants). For 500 $-\mathrm{keV}$ cold implants, it can also be observed that an increase in the amount of displaced Ga can be observed in the surface region (which is not observed for the $200-\mathrm{keV}$ implant due to the high level of damage in that region and/or the resolution of RBS).

The SIMS spectra that are shown in Fig. 2 present the $\mathrm{Mg}$ profiles that are formed in GaN following implantation

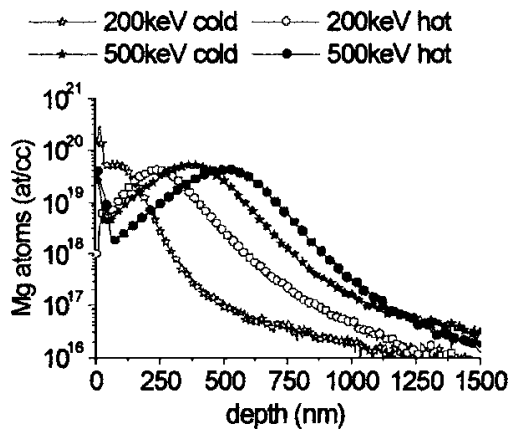

FIG. 2. SIMS spectra obtained from GaN samples implanted with $\mathrm{Mg}$ ions at different energies and different temperatures. 


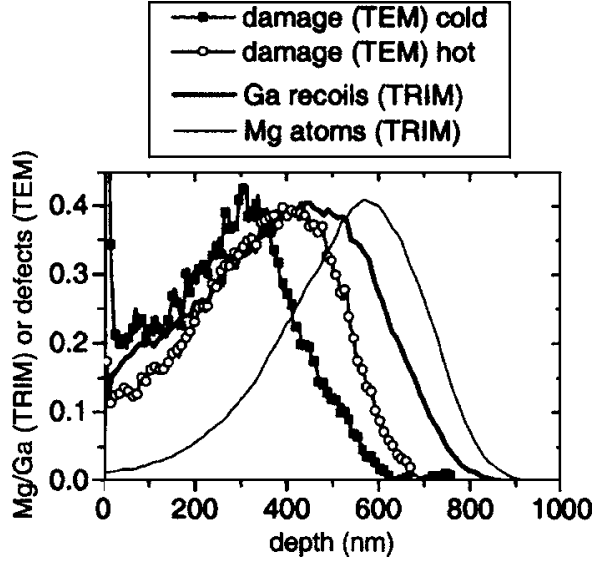

FIG. 3. The simulated $\mathrm{Mg}$ range and the $\mathrm{Ga}$ recoil distribution in $\mathrm{GaN}$ for 500-keV Mg implants (as predicted with TRIM). N.B. the intensity profiles from XTEM have been extracted (from Fig. 4), and have also been incorporated into the figure.

(at 200 and $500 \mathrm{keV}$ ) at hot and cold temperatures. It can be seen that for both implant energies the $\mathrm{Mg}$ range in the solid is reduced for cold implants, and the peak position is shifted towards the surface with respect to hot implants. No additional contaminated surface layers have been observed on the surface (with SIMS, RBS, and XTEM) that could cause this apparent shift in profile position.

If we compare the damage peak position (from RBS) and the Mg peak position (from SIMS), then for hot implantation the $\mathrm{Mg}$ peak is deeper than the damage peak. In contrast, for the cold implants the $\mathrm{Mg}$ peak position is shallower than the damage peak position. It is also interesting to note that the shift in the damage peak position as a function of the implant temperature is not the same as the shift in the $\mathrm{Mg}$ peak position as a function of the implant temperature.

Implant simulations in $\mathrm{GaN}$ have been performed with TRIM. It was not possible to consider the implant temperature during the simulation, and the material constants used for the ion stopping and the recoil formation are applicable to roomtemperature conditions. It can be seen in Fig. 3 that theoretically, the $\mathrm{Mg}$ comes to rest within the solid at a depth beyond the Ga recoil distribution, which is in line with the observations made above for the hot implants.

The simulations do not match exactly with the measured profiles, and this could be a real consequence of the temperature (and the rate of dynamic annealing, which is not considered during TRIM) and/or the constants for the material properties used $\left(\rho=6.15 \mathrm{~g} / \mathrm{cm}^{3}\right.$, displacement energy of $\mathrm{N}$ in $\mathrm{GaN}$ is $28 \mathrm{eV}$ and the displacement energy of $\mathrm{Ga}$ in $\mathrm{GaN}$ is $25 \mathrm{eV}$, N.B. the latter two values have been suggested by TRIM). The predicted range of 500-keV Mg ions implanted into $\mathrm{GaN}$ at room temperature is $580 \mathrm{~nm}$, and the $\mathrm{Mg}$ range measured experimentally with SIMS is $380 \mathrm{~nm}$ for cold implants and $510 \mathrm{~nm}$ for hot implants. The predicted damage peak position for 500-keV Mg implanted into $\mathrm{GaN}$ at room temperature is $465 \mathrm{~nm}$, and the damage peak position measured with RBS is $420 \mathrm{~nm}$ for cold implants and $445 \mathrm{~nm}$ for hot implants. For all case scenarios (including 200-keV implants-results not shown), the implant profiles formed in hot samples match more closely with the simulated profiles.

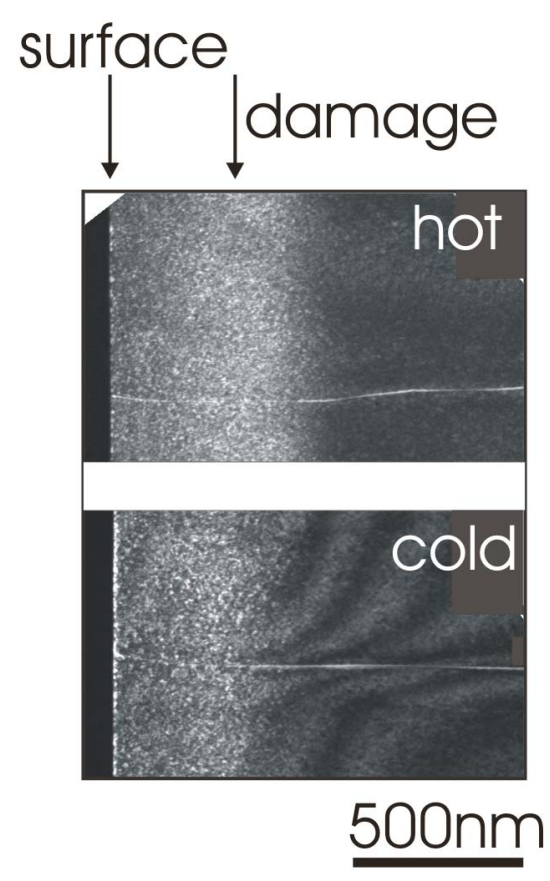

FIG. 4. Weak-beam dark-field cross-section TEM images taken from a 500-keV Mg-implanted GaN sample (implanted at hot and cold temperatures). The horizontal feature is a crystal crack coming to the GaN surface.

Weak-beam dark-field XTEM has been performed upon samples implanted at $500 \mathrm{keV}$. The RBS data give an indication of the displaced $\mathrm{Ga}$ in the solid, while the SIMS data give a measure of the $\mathrm{Mg}$ profile in the solid. TEM gives a measure of both $\mathrm{Mg}$ and $\mathrm{Ga}$ defects, as well as any other defects that reside within the solid, i.e., $\mathrm{N}$ defects. In Fig. 4, the difference in the damage distribution for samples implanted with 500-keV Mg ions at hot and cold temperatures is evident. The defect range in the cold sample appears shallower, while the hot sample shows an extended defect distribution which, although does not match in depth with the RBS (Ga disorder only) data, is in agreement with the trends observed.

It is interesting to note that the differences in the TEM damage profile (particularly the peak position and the end of range damage), as a function of the implant temperature, appear much more apparent than for the RBS data at this implant energy. This is an indication that we are observing different defect species. The intensity damage profile has been extracted from these TEM images (shown in Fig. 3). The damage peak positions from the TEM analysis $(310 \mathrm{~nm}$ for cold and $410 \mathrm{~nm}$ for hot implants) do not match with the RBS results (420 nm for cold and $445 \mathrm{~nm}$ for hot implants). This difference, as indicated above, is believed to be caused by the sensitivity of the TEM analysis technique to different types of defect in the samples (such as clusters and $\mathrm{N}$ defect complexes that are not detectable with RBS). The TEM damage profiles appear shallower than the RBS profiles, and it is possible that the $\mathrm{Ga}$ atoms detected at the end of range with RBS may not be visible to TEM. However, a marked difference in the damage can be observed at the surface with TEM for cold implants, which is in agreement with the RBS measurements when an increase in the interstitial Ga is observed.

The stability of the implant damage and the Mg distri- 

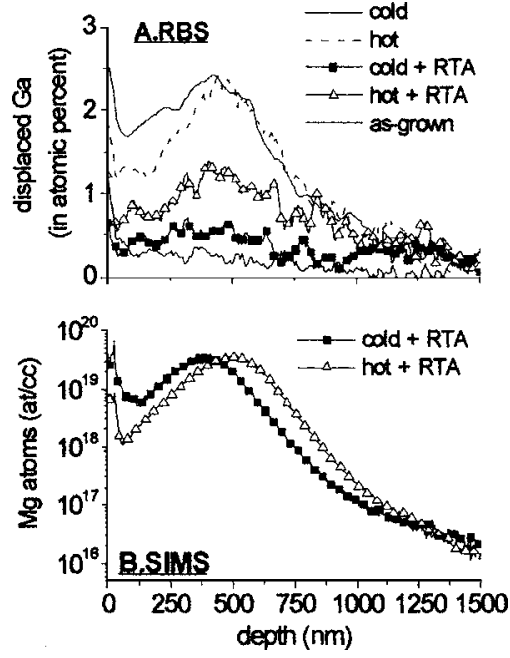

FIG. 5. RBS (a) and SIMS (b) spectra obtained from GaN samples implanted with 500-keV Mg ions at different temperatures, with and without a postimplant thermal process (at $1000{ }^{\circ} \mathrm{C}$ for $20 \mathrm{~s}$ in $\mathrm{N}_{2}$ ).

bution within the solid has been assessed with a postimplant high-temperature $\left(1000{ }^{\circ} \mathrm{C}\right)$ anneal. Damage annealing does occur at this temperature [Fig. 5(a)], and the rate of damage removal shows a dependence upon the implant temperature. For hot implants post-RTA, a damage peak can be observed which is aligned with the as-implanted damage peak. The damage removal process in the sample implanted at cold temperature is much more effective than in the sample implanted at the higher temperature. For cold implants the residual damage that remains post-RTA is comparable to the as-grown level. The increased disorder observed within the surface region for cold samples has been removed during RTA. The SIMS profiles in Fig. 5(b) indicate that the $\mathrm{Mg}$ atoms remain stationary and no diffusion is observed.

\section{DISCUSSION}

The dependence of the damage formation and the dopant range in $\mathrm{GaN}$ upon the substrate temperature during $\mathrm{Mg}$ implantation has been investigated. Clear evidence has been provided with RBS, SIMS, and TEM analyses that the substrate temperature during the implantation process affects the damage formation (volume, complexity, and distribution in the solid) and the Mg profile. The techniques employed have clearly indicated that the damage range and the $\mathrm{Mg}$ range in the solid are reduced for cold implantation. It was also demonstrated that the damage formed in GaN during hightemperature implantation is more stable in nature during furnace annealing when compared to samples implanted at cold temperatures.

These observations can be explained by a change in the rate of the dynamic defect annealing caused by the change in the implant temperature. For cold implants the rate of annealing is reduced and the amount of recoil recombination/ defect annihilation during ion irradiation is lowered in comparison to hot implants. The lack of annealing (or reduced annealing) in cold implants leads to an increase in the rate of damage buildup with respect to the sample implanted at an elevated temperature. As the damage builds up for cold im- plants, the rate of ion stopping in the solid is increased (due to an increase in the number of scattering centers), and the $\mathrm{Mg}$ range is subsequently reduced. In turn, the reduction in $\mathrm{Mg}$ range inherently reduces the damage profile. As far as the authors are aware, this dopant profile dependence upon the implant temperature has not been reported elsewhere. At higher-temperature implants, although the rate of dynamic annealing is raised, the likelihood of a complex defect being formed is also increased. As a result, the damage which is formed is more stable in nature when compared to the damage formed in samples implanted at cold temperatures. For implantation performed at cold temperatures, although more defects remain post-implant, they are simpler in nature. These trends are in line with the arguments proposed in Refs. $2-4$.

The general results collected and the trends observed have been discussed and an argument based on defect dynamic annealing has been proposed. However, the authors feel that two observations warrant further discussion, namely, the fact that the $\mathrm{Mg}$ and Ga disorder peak positions change relatively to each other as a function of the implant temperature, and the fact that the shift in $\mathrm{Mg}$ peak position as a function of temperature does not match the shift in the $\mathrm{Ga}$ disorder.

We have measured the displaced Ga distributions and the $\mathrm{Mg}$ distributions as a function of the implant temperature. For fixed implant doses, the position of the $\mathrm{Mg}$ peak in the hot samples is always beyond the Ga damage peak position (as also predicted with TRIM), and for cold implants the $\mathrm{Mg}$ peak position always lies below the Ga damage peak position. As discussed above, due to the level of dynamic defect annealing, the rate of ion stopping is increased for cold implants, and hence the number of displaced atoms that remain in the solid for each individual ion postirradiation will be raised when compared to hot implants. We propose that this extra generation of displaced atoms for the cold implants explains why the $\mathrm{Ga}$ disorder always extends beyond the $\mathrm{Mg}$ profile (and also why the Ga disorder at the surface is higher for cold implants) when compared to hot implants.

The change in the disordered Ga profile as a function of temperature is not matched by the same change in the $\mathrm{Mg}$ profile. This suggests that although the trends are consistent (as they move in the same direction), the magnitude of the movement is not constant. This suggests that the Ga and the $\mathrm{Mg}$ transport properties in the solid are different.

\section{CONCLUSION}

The radiation damage formation process and the $\mathrm{Mg}$ range in $\mathrm{GaN}$ during $\mathrm{Mg}$ ion implantation depend upon the implant temperature. For cold implantation $\left(-150{ }^{\circ} \mathrm{C}\right)$, when the level of defect dynamic annealing is reduced, the $\mathrm{Mg}$ range is reduced in the solid due to the increase in disorder (scattering centers). As a consequence, the damage range is therefore reduced. The damage stability during postimplant furnace annealing $\left(1000{ }^{\circ} \mathrm{C}\right)$ has also been investigated. It has been shown that due to increased levels of defect move- 
ment (annealing) in hot $\left(+300{ }^{\circ} \mathrm{C}\right)$ implants, the likelihood of a stable defect complex forming is raised, and hence more stable damage is observed in these samples.

\section{ACKNOWLEDGMENTS}

The authors wish to thank J. Yan, M. Kappers, and C. Humphreys (Department of Materials Science and Metallurgy, University of Cambridge, UK) for providing GaN material. SIMS has been performed at LSA Ltd. (UK). The project has been supported by EPSRC, Project No. GR/ R70842/01.
${ }^{1}$ J. K. Sheu and G. C. Chi, J. Phys.: Condens. Matter 14, R657 (2002). ${ }^{2}$ S. Whelan, J. A. Van den Berg, S. Zhang, D. G. Armour, and R. D. Goldberg, Appl. Phys. Lett. 76, 571 (2000).

${ }^{3} \mathrm{~S}$. Ahmed, B. J. Sealy, and R. Gwilliam, Proceedings of the 14th International Conference on Ion Implantation Technology, Piscataway, New Jersey, 22-27 September 2002 (IEEE, New York, 2003), pp. 60-63.

${ }^{4}$ C. Liu, A. Wenzel, K. Volz, and B. Rauschenbach, Nucl. Instrum. Methods Phys. Res. B 148, 396 (1999).

${ }^{5}$ J. F. Ziegler, Handbook of Ion Implantation Technology (North-Holland, Amsterdam 1992).

${ }^{6}$ G. Eftekhari, Semicond. Sci. Technol. 6, 193 (1991).

${ }^{7}$ W. K. Chu, J. W. Mayer, and M. A. Nicolet, Backscattering Spectrometry (Academic, New York, 1978)

${ }^{8}$ N. P. Barradas, C. Jeynes, and R. P. Webb, Appl. Phys. Lett. 71, 291 (1997). 